\title{
Periodic Mechanical Stress Stimulates Cav- 1-Dependent IGF-1R Mitogenic Signals in Rat Chondrocytes Through ERK1/2
}

\author{
Kewei Ren ${ }^{\mathrm{a}} \quad$ Jilei Tang ${ }^{\mathrm{b}} \quad$ Xuefeng Jiang $^{\mathrm{a}} \quad$ Huiqing Sun ${ }^{\mathrm{a}} \quad$ Luming Nong $^{\mathrm{c}}$ \\ Nan Shen ${ }^{d}$ Yanqing Gue
}

aDepartment of Orthopedics, the Affiliated Jiangyin Hospital of Southeast University Medical School, Jiangyin, 'Department of Orthopedics, Qidong People's Hospital, Nantong, 'Department of Orthopedics, the Affiliated Changzhou No.2 People's Hospital with Nanjing Medical University, Changzhou, 'Department of Clinical Pharmacy, the Affiliated Jiangyin Hospital of Southeast University Medical School, Jiangyin, eDepartment of Orthopedics, Nanjing First Hospital, Nanjing Medical University, Nanjing, China

\section{Key Words}

Periodic mechanical stress - Chondrocyte proliferation - Non-integrin mechanosensors $•$ Cav$1 \cdot$ IGF-1R

\begin{abstract}
Background/Aims: The biological effects of periodic mechanical stress on the mitogenesis of chondrocytes have been studied extensively over the past few years. However, the mechanisms underlying the ability of chondrocytes to sense and respond to mechanical stimuli remain to be determined. In the current study, we analyzed the mechanisms by which periodic mechanical stress is translated into biochemical signals and verified the key role of non-integrin mechanosensors including Caveolin-1 (Cav-1), and insulin-like growth factor-1 receptor (IGF-1R) in chondrocyte proliferation. Methods: Two steps were undertaken in the experiment. In the first step, the cells were maintained under static conditions or periodic mechanical stress for $0 \mathrm{~h}$ and $1 \mathrm{~h}$ prior to Western blot analysis. In the second step, the cells were pretreated with short hairpin RNA (shRNA) targeted to Cav-1 or IGF-1R or control scrambled shRNA. Moreover, they were pretreated with their selective inhibitors methyl $\beta$-cyclodextrin (MCD) or Linsitinib (OSI-906). They were maintained under static conditions or periodic mechanical stress for $1 \mathrm{~h}$ prior to Western blot analysis, and for 3 days, $8 \mathrm{~h}$ per day, prior to direct cell counting and CCK-8 assay, respectively. Results: Periodic mechanical stress significantly induced sustained phosphorylation of Cav-1 at Tyr ${ }^{14}$ and IGF-1R at Tyr ${ }^{1135 / 1136}$. Proliferation was inhibited by pretreatment with Cav-1 inhibitor MCD and by shRNA targeted to Cav-1 in chondrocytes in response to periodic mechanical stress. Meantime, MCD and shRNA targeted to Cav-1 also attenuated IGF-1R, and extracellular signal-regulated kinase (ERK)1/2 activation. In addition, inhibiting IGF-1R activity by Linsitinib and shRNA targeted to


IGF-1R abrogated chondrocyte proliferation and phosphorylation level of ERK1/2 subjected to periodic mechanical stress, while the phosphorylation site of Cav-1 was not affected. Conclusion: These findings collectively suggested that periodic mechanical stress promoted chondrocyte proliferation through Cav-1-IGF-1R-ERK1/2.

(C) 2018 The Author(s)

Published by S. Karger AG, Basel

\section{Introduction}

Periodic mechanical stress is advantageous to simulate in vivo physiological conditions for promoting chondrocyte proliferation [1, 2]. However, few studies have addressed the mechanisms underlying the translation of such mechanical inputs into biochemical signaling events within the chondrocyte lineage.

As is known to all, cell perception and response to extracellular stimuli depend on a variety of receptors on the surface of the cell membrane. Therefore, elucidation of the mechanosensors is essential for determining the signal transduction mechanism by which periodic mechanical stress promotes chondrocyte proliferation. Insulin-like growth factor-1 receptor (IGF-1R) is a cell-surface, membranespanning protein, with intrinsic tyrosine kinase activity. IGF-1R is involved in controlling the activation of downstream signaling proteins and these pathways can mediate multiple cellular biological activity and functions $[3,4]$. IGF-1R, as a potential mechanosensor, is known to be tightly linked to non-integrinassociated mechanotransduction [5, 6]. A study by Fu confirmed that cyclic mechanical stretch-enhanced myoblast proliferation was dependent on activated IGF-1R [7]. IGF-1R has been shown to play a vital role in the modulation of chondrocyte activity and functions following chemical stimuli [8]. However, it is presently obscure whether IGF-1R is involved in modulating mechanical stress-initiated mitogenic effects in chondrocytes. Moreover, the nature of the relationship between IGF-1R and downstream extracellular signal-regulated kinase (ERK)1/2 MAPKS remains to be elucidated.

Caveolins are a family of integral membrane proteins that are the principal components of caveolae membranes. Accumulating evidence supports a critical role of Caveolin-1 (Cav-1) in responses to various stimuli from the cellular peripheral environment that affect cellular biological processes [9-11]. Several researchers have established that Cav-1, as a non-integrin mechanosensor, can be activated by mechanical stimuli besides biochemical stimulation, including growth factors $[12,13]$. Chai and co-workers established that activation of Cav-1 is essential for the generation of vasodilators of mouse small coronary arteries under shear stress [14]. Yang and collaborators reported that fluid shear stress promoted breast cancer motility, invadopodia formation and metastasis in vivo via mechanosensitive Cav-1 activation [15]. However, no study demonstrated the mitogenic effects of Cav-1 signals in chondrocytes in response to mechanical stimulation.

Activated Cav-1 can interact with and activate cell-surface growth factor receptors and other kinases to form signaling aggregates, and anchor potential downstream signaling molecules to the cell membrane, activating intracellular signaling cascades [16, 17]. Zhang et al. demonstrated dependence of mechanical stretch-induced activation of EGFR signals on Cav-1 in mesangial cells [18]. Yang and colleagues proved that advanced glycation end products (AGEs) stimulated Cav-1 and IGF-1R to form signaling complex that promoted the differentiation of 3T3-L1 preadipocytes, and Cav-1 led to the activation of IGF-1R in this system [19]. However, little is known about the association between Cav-1 and IGF-1R in chondrocytes subjected to mechanical stimuli. Elucidation of the nature of any functional association among Cav-1, IGF-1R, and ERK1/2 in chondrocytes is essential in this system.

This study aimed to determine the mechanisms by which periodic mechanical stress is translated into biochemical signals, and verified the pivotal role of non-integrin mechanosensors including Cav-1, and IGF-1R in chondrocyte proliferation. Additionally, association among of Cav-1, IGF-1R, and ERK1/2 in this system was analyzed to establish the mechanotransduction pathways and link these signals into mitogenic cascades. 


\section{Cellular Physiology Cell Physiol Biochem 2018;48:1652-1663 \begin{tabular}{l|l} 
and Biochemistry Published 10.1159/000492288 & (c) 2018 The Author(s). Published by S. Karger AG, Basel \\
www.karger.com/cpb
\end{tabular}}

Ren et al.: Cav-1-Dependent IGF-1R Mitogenic Signals Through ERK1/2

\section{Materials and Methods}

\section{Materials}

Two-week-old Sprague-Dawley rats of either sex were provided by the Animal Center of Nanjing Medical University. Dulbecco's modified Eagle's medium-nutrient mixture F-12, fetal bovine serum, trypsin, collagenase II, and anti-collagen monoclonal antibody II were purchased from Gibco (USA). Type II collagen and MCD were purchased from Sigma (USA). Cell counting kit-8 (CCK-8) was purchased from Beyotime Institute of Biotechnology (China). Anti-Cav-1, anti-phospho-Cav-1 (Tyr ${ }^{14}$ ), anti-IGF-1R $\beta$, anti-phosphoIGF-1R $\beta$ (Tyr ${ }^{1135 / 1136}$ ), anti-ERK1/2, anti-phospho-ERK1/2 $\left(\mathrm{Thr}^{202} / \mathrm{Tyr}^{204}\right)$, horseradish peroxidase-goat anti-rabbit IgG, and enhanced chemiluminescence (ECL) assay kit were supplied by CST Co., Ltd. (USA). Linsitinib (OSI-906), Cav-1 shRNA lentiviral particles, IGF-1R shRNA lentiviral particles, Control shRNA lentiviral particles, and Polybrene were purchased from Santa Cruz (USA).

A cell incubator (Hereus BB 5060), air-tight cell culture device and reciprocating pressure pump, barrier-type pressure transducer and inversion microscope equipped with camera system were bought from Hereus (Germany), Taixing Experimental Instrument Factory (China), Tianjin Plastics Research Institute (China) and Olympus (Japan), respectively.

\section{Methods}

Cell culture. Chondrocytes were harvested using the method described in our previous study [20]. The cells were purified by repeated adherence, and the morphology was observed under an inverted phase contrast microscope by staining for collagen type II according to the conventional ABC method. The cells of the second generation were seeded on a glass slide $\left(25 \times 25 \mathrm{~mm}^{2}\right)$ coated with type II collagen at a density of $10^{5}$. Experiments were performed when the cells were approximately $70-80 \%$ confluent.

Inhibitors. MCD was a specific inhibitor against Cav-1, and Linsitinib was a specific inhibitor against IGF-1R. MCD was dissolved in DMEM, and Linsitinib was dissolved in anhydrous dimethylsulfoxide (DMSO) to form 1000 concentrated solution. All inhibitors were aliquoted and stored at $-20^{\circ} \mathrm{C}$. Each concentrated solution was diluted by $1000 \times$ immediately prior to use; Linsitinib pretreatment groups contained $0.1 \%$ $(\mathrm{v} / \mathrm{v})$ DMSO, which was the concentration used for the control group. The cells were pretreated with Linsitinib $(1 \mu \mathrm{M})$, or an equivalent amount of DMSO $(0.1 \% \mathrm{v} / \mathrm{v})$ for $1 \mathrm{~h}$. The remaining cells were pretreated with MCD (1 mM), or an equivalent amount of DMEM for $1 \mathrm{~h}$.

Construction of a periodical mechanical stress field. A periodic stress field of perfusion culture system with adjustable stress intensity and frequency was built by connecting the reciprocating intensifier pump to the air-tight cell culture device through a barrier-type pressure transducer, as previously described [21]. The reciprocating intensifier pump was mainly composed of a compression power device, a piston, and an air-tight pressure chamber filled with a fluid. The compression power device created a mechanical reciprocating motion by connecting with the piston and squeezing the fluid of the air-tight pressure chamber to generate periodic hydraulic pressure, acting on the pressure transducer through the input terminus. The pressure transducer was composed of a diaphragm and a pressure monitor. The diaphragm was located in the middle of the pressure transducer and separated the bilateral fluids of the input terminus and the output terminus, which transmitted the periodic hydraulic pressure produced by compressive deformation of the reciprocating compression pump to the air-tight cell culture device filled with the cell culture medium. The pressure monitor was located in the output terminus of the pressure transducer, permitting monitoring and regulation of hydraulic pressure in real time to maintain a constant intensity and frequency. Hydraulic pressure periodically acted on chondrocytes of the second generation by inoculating them onto the slide in the air-tight cell culture device.

The pressure in this system ranged from 0 to $300 \mathrm{kPa}$ and the frequency from 0 to $1 \mathrm{~Hz}$. Earlier, it was shown that rabbit chondrocytes subjected to stress varying from 0 to $200 \mathrm{kPa}$ at $0.1 \mathrm{~Hz}$ yielded tissueengineered cartilage of the best quality [22, 23]. Accordingly, a pressure range of $0-200 \mathrm{kPa}$ and $0.1 \mathrm{~Hz}$ frequency were used in the current study. 


\section{Cellular Physiology Cell Physiol Biochem 2018;48:1652-1663 \begin{tabular}{l|l|l}
\hline DOI: 10.1159/000492288 & $\begin{array}{l}\text { () 2018 The Author(s). Published by S. Karger AG, Basel } \\
\text { www.karger.com/cpb }\end{array}$
\end{tabular}}

Experimental grouping. Two steps were undertaken in the experiment. In the first step, the cells were maintained under static conditions or periodic mechanical stress for $0 \mathrm{~h}$ and $1 \mathrm{~h}$ prior to Western blot analysis. In the second step, the cells were pretreated with shRNA targeted to Cav-1 or IGF-1R or control scrambled shRNA. Moreover, they were pretreated with MCD $(1 \mathrm{mM})$ or Linsitinib $(1 \mu \mathrm{M})$. They were maintained under static conditions or periodic mechanical stress for $1 \mathrm{~h}$ prior to Western blot analysis, and for 3 days, $8 \mathrm{~h}$ per day, prior to direct cell counting and CCK-8 assay, respectively.

All groups of cells involved in the experiments were incubated at $37^{\circ} \mathrm{C}$ in the incubator with $5 \% \mathrm{CO}_{2}$.

Western blot analysis. Total protein was prepared using radioimmunoprecipitation assay (RIPA) buffer, and Western blot analyses were performed as previously described [21]. Total protein was prepared using RIPA buffer and analyzed for protein concentration using Bradford assay. Protein samples were resolved by sodium dodecyl sulfate polyacrylamide gel electrophoresis and transferred onto nitrocellulose membranes. Following blocking for $1 \mathrm{~h}$ with 5\% milk in Tris-buffered saline and Tween 20, the membranes were incubated with antibodies (1:1000 dilution for three antibodies) overnight at $4^{\circ} \mathrm{C}$. The blots were then incubated with horseradish peroxidase-conjugated secondary antibody at room temperature for $1 \mathrm{~h}$ and developed colors with ECL. The results were scanned using Gel Imaging System (UVP Company, USA) and measured using Gel-Pro Analyzer software (Media Cybernetics, USA).

Proliferation Studies. Proliferation studies were assessed by two different methods: direct cell counting and CCK-8 assay.

Direct cell counting. The cells were trypsinized and counted as previously described [24]. The cell number was determined by counting each piece of glass slide independently. Each group included six random pieces of glass slides and the experiments were repeated five times.

CCK-8 assay. Cell proliferation was determined by using CCK-8 solution according to the manufacturer's instruction as previously described [2]. The cells were kept in five 96-well plates ( $n=5) ; 10 \mu \mathrm{L}$ of CCK-8 solution was added to each well and incubated for $4 \mathrm{~h}$ at $37^{\circ} \mathrm{C}$. The absorbance of each well was finally determined at $450 \mathrm{~nm}$ using a microplate reader.

Statistical analysis. Statistical analyses were performed using SPSS 14.0 software (SPSS, IL, USA), and the results were expressed as mean \pm standard deviation. Student's unpaired $t$ tests were used to determine the statistical significance. A $P$ value of 0.05 was considered to be significant.

\section{Results}

Effects of Periodic Mechanical Stress on Cav-1 and IGF-1R Phosphorylation

The phosphorylation levels of Cav-1 and IGF-1R at $1 \mathrm{~h}$ increased in the pressure group compared to the non-pressure group $(P<0.05$ for each, Fig. $1, n=5)$.

Cav-1 and IGF-1R Are Required for Periodic Mechanical Stress-initiated Chondrocyte Proliferation

Cav-1 and IGF-1R inhibition with their selective inhibitors (MCD and Linsitinib) and targeted shRNA both blocked periodic mechanical stress-induced chondrocyte proliferation compared with chondrocytes in the control groups in response to periodic mechanical stress $(P<0.05$ for each, Figs. 2 and $3, n=5)$.

IGF-1R Inhibition Prevents Periodic Mechanical Stress-induced ERK1/2 Phosphorylation

IGF-1R inhibition by Linsitinib pretreatment and shRNA targeted to IGF-1R both abrogated the levels of ERK1/2 phosphorylation in chondrocytes under conditions of mechanical stress $(P<0.05$ for each, Figs. 4 and $5, n=5)$. 


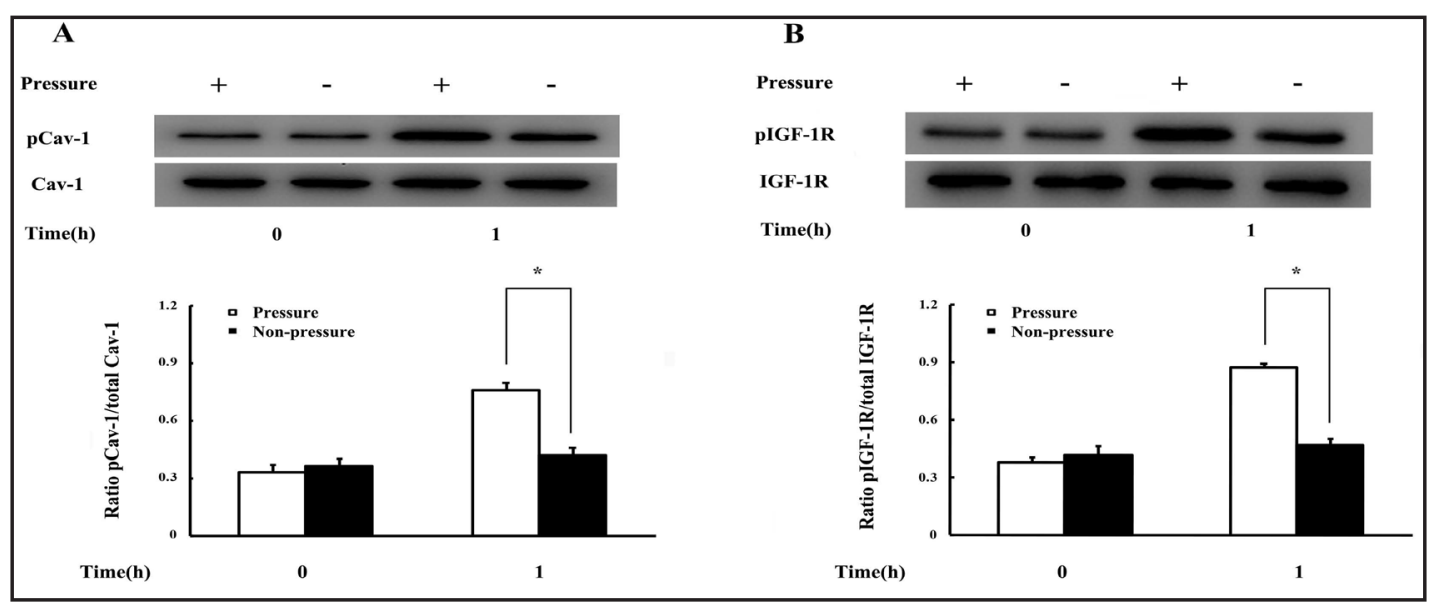

Fig. 1. Effects of periodic mechanical stress on the phosphorylation of Cav-1 and IGF-1RRat chondrocytes were cultured in vitro for $0 \mathrm{~h}$ and $1 \mathrm{~h}$ with or without periodic mechanical stress. The expression and phosphorylation levels of Cav-1 and IGF-1R were detected by Western blotting. The total amount of protein served as a control. Gray values are represented with a histogram ( $n=5, * \mathrm{P}<0.05$ for each). The aforementioned images are representative results of Western blotting. The phosphorylation levels of Cav1-Tyr ${ }^{14}$ and IGF-1R-Tyr ${ }^{1135 / 1136}$ in the pressure groups significantly increased relative to those of the nonpressure groups ( $\mathrm{n}=5, \mathrm{P}<0.05$ for each; Student's unpaired t test).

Fig. 2. Effects of Cav-1 and IGF-1R on chondrocyte proliferation under conditions of periodic mechanical stress (inhibitors)After pretreatment with DMEM or Cav-1 selective inhibitor MCD (1 $\mathrm{mM})$, or DMSO or IGF-1R selective inhibitor Linsitinib $(1 \mu \mathrm{M})$, rat chondrocytes were cultured for 3 days under static conditions or conditions of periodic mechanical stress $8 \mathrm{~h}$ per day prior to proliferation studies. Chondrocyte proliferation was analyzed using direct cell counting (A) and CCK8 assay (B). Chondrocyte proliferation results are shown in the histogram $(n=5$, $* \mathrm{P}<0.05$ for each). Chondrocyte proliferation in the selective

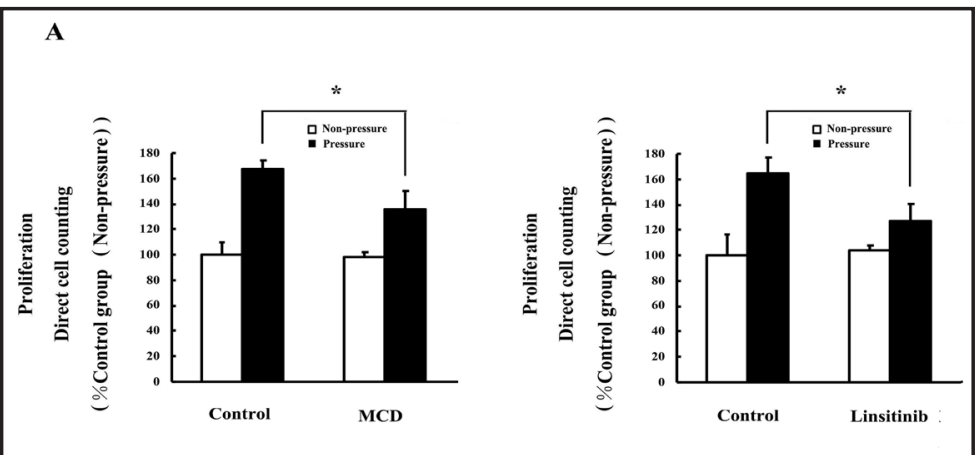

$\mathbf{B}$

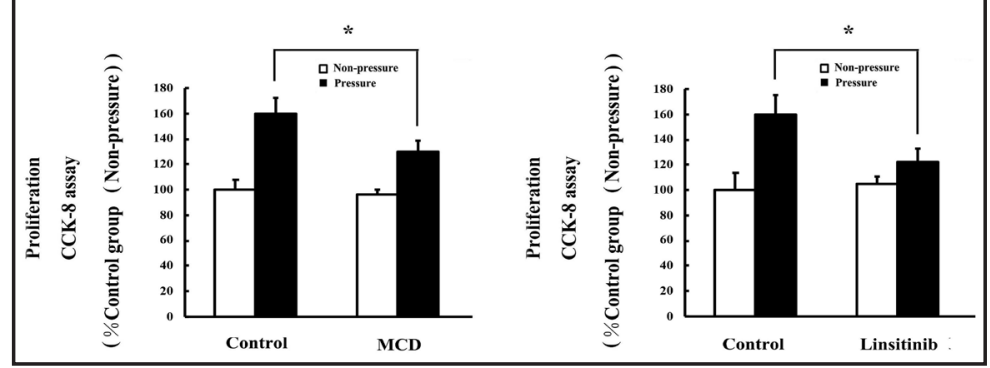

inhibitor pretreatment groups significantly decreased relative to that in the control groups under conditions of periodic mechanical stress. ( $n=5, P<0.05$ for each; Student's unpaired t test). 


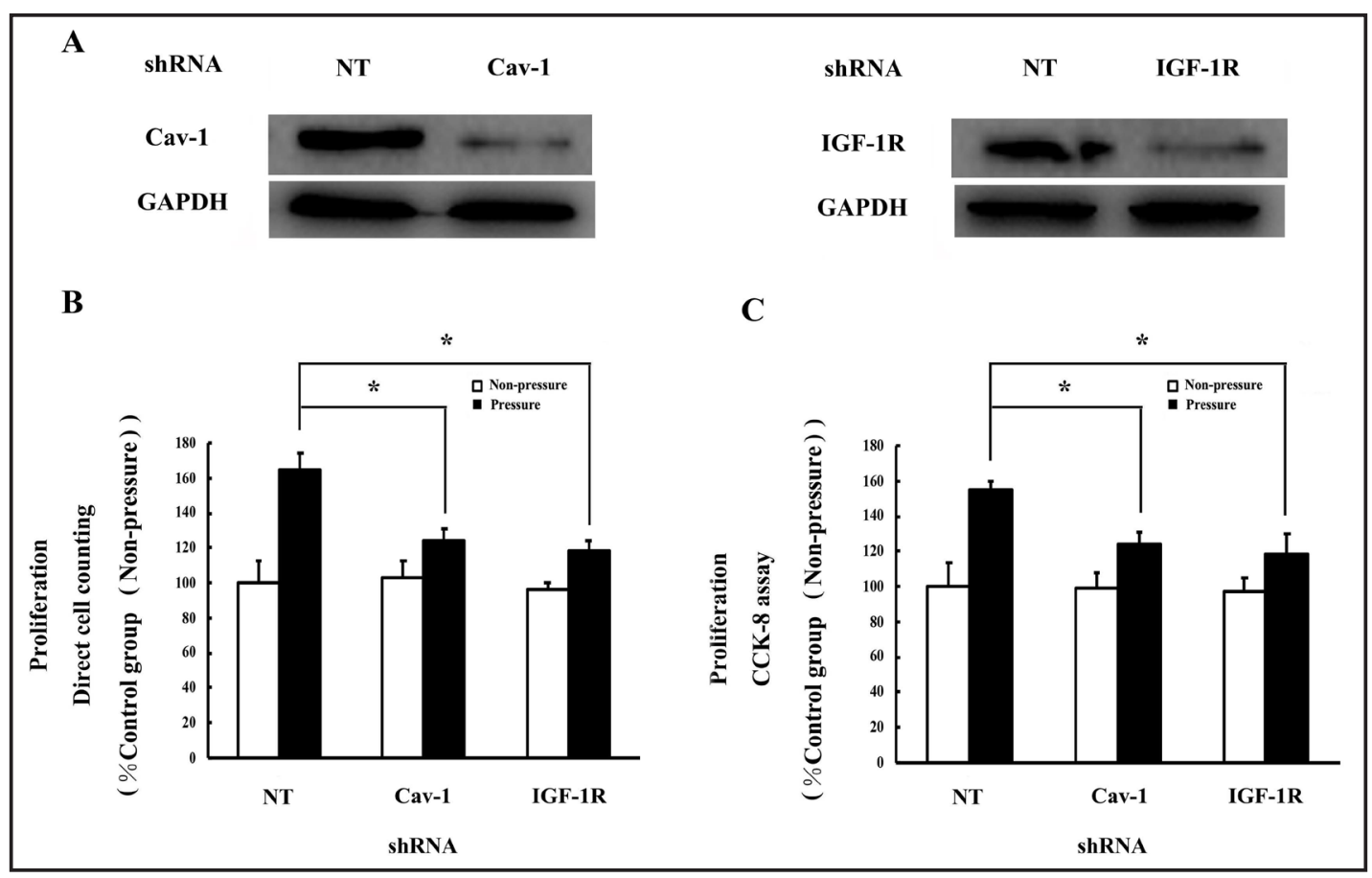

Fig. 3. Effects of Cav-1 and IGF-1R on chondrocyte proliferation under conditions of periodic mechanical stress (shRNA)Chondrocytes were transfected with shRNA targeted to Cav-1 or IGF-1R or with nontargeting sequences prior to lysis and Western blotting for Cav-1 and IGF-1R proteins. Transfection with shRNA for Cav-1 and IGF-1R achieved about a 50\% reduction in Cav-1 and IGF-1R protein levels, respectively (A). After pretreatment with control or Cav-1 shRNA or IGF-1R shRNA, rat chondrocytes were cultured for 3 days under static conditions or conditions of periodic mechanical stress $8 \mathrm{~h}$ per day prior to proliferation studies. Chondrocyte proliferation was analyzed using direct cell counting (B) and CCK-8 assay (C). Chondrocyte proliferation results are shown in the histogram ( $\mathrm{n}=5,{ }^{*} \mathrm{P}<0.05$ for each). Chondrocyte proliferation significantly decreased in the shRNA pretreatment groups relative to that in the control groups under conditions of periodic mechanical stress ( $n=5, P>0.05$ for each; Student's unpaired t test).

\section{IGF-1R Is Not Required for Periodic Mechanical Stress-induced Cav-1 Phosphorylation}

Inhibition of IGF-1R with the selective inhibitor Linsitinib and shRNA targeted to IGF-1R did not reduce periodic mechanical stress-induced Cav-1 activation in chondrocytes under mechanical conditions $(P>0.05$ for each, Figs. 4 and $5, n=5)$.

Cav-1 Is Required for Periodic Mechanical Stress-induced IGF-1R and ERK1/2 Phosphorylation

Pretreatment with Cav-1 selective inhibitor MCD and shRNA targeted to Cav-1 both attenuated periodic mechanical stress-induced IGF-1R and ERK1/2 phosphorylation in chondrocytes under conditions of periodic mechanical stimulation $(P<0.05$ for each, Figs. 6 and $7, n=5)$.

\section{Discussion}

Periodic mechanical stress induction of chondrocyte proliferation may be mediated through a process of mechanotransduction, converting physical forces into biochemical signals and integrating these signals into cellular responses. Our present findings demonstrated that Cav-1 and IGF-1R, as nonintegrin mechanosensors, mediate the ERK1/2 mitogenic signaling cascade in chondrocytes in response to periodic mechanical stress. 
Fig. 4. Effects of IGF-1R on the expression and phosphorylation of Cav-1 and ERK1/2 under conditions of periodic mechanical stress (Linsitinib) After pretreatment with DMSO or IGF-1R inhibitors Linsitinib, rat chondrocytes were cultured in vitro for $1 \mathrm{~h}$ with or without periodic mechanical stress. The expression and phosphorylation levels of IGF-1R, Cav-1, and ERK1/2 were detected by Western blotting. The total amount of each protein served as a control. Gray values are represented with a histogram ( $\mathrm{n}=5,{ }^{*} \mathrm{P}<0.05$ for each). The aforementioned images are representative results of Western blotting. The phosphorylation levels of IGF-1-Tyr ${ }^{1135 / 1136}$ and ERK1/2-Thr ${ }^{202} / \mathrm{Tyr}^{204}$ in the Linsitinib pretreatment group

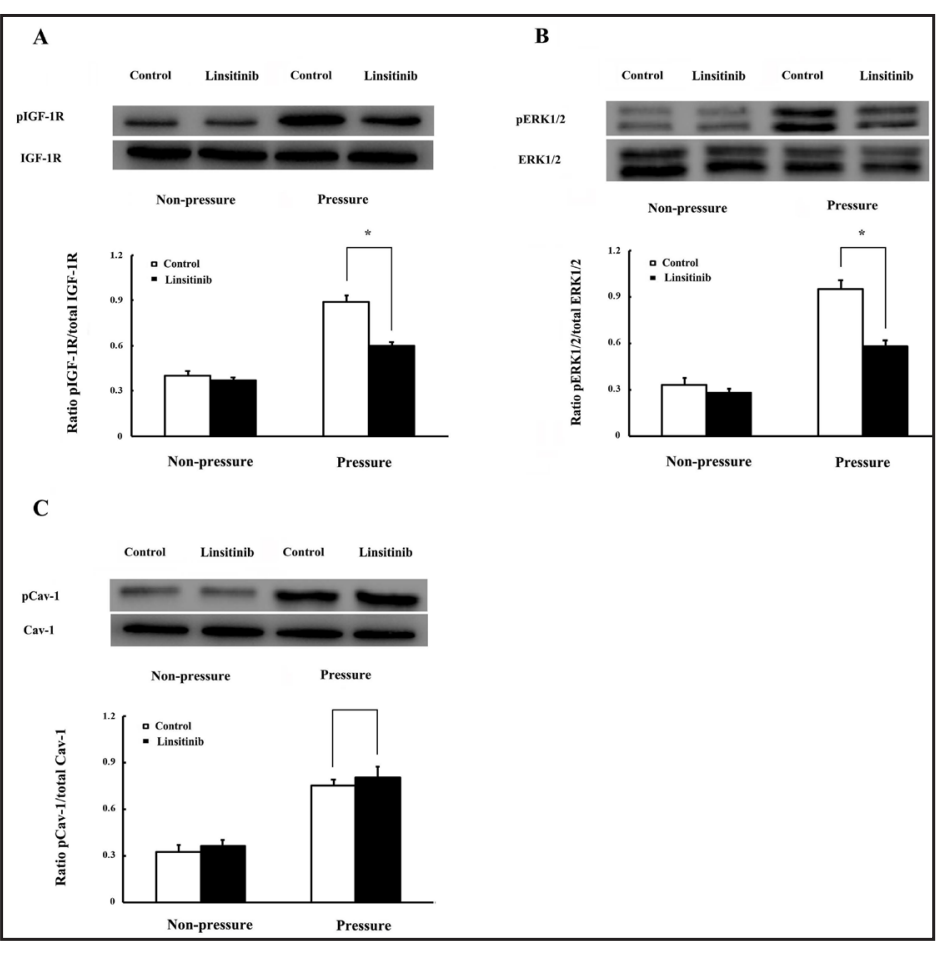
significantly decreased relative to those of the control group under conditions of periodic mechanical stress ( $n=5, P<0.05$ for each; Student's unpaired $t$ test), whereas the activation of Cav-1-Tyr ${ }^{14}$ in the Linsitinib pretreatment group was not affected ( $\mathrm{n}=5, \mathrm{P}>0.05$ for each; Student's unpaired t test).

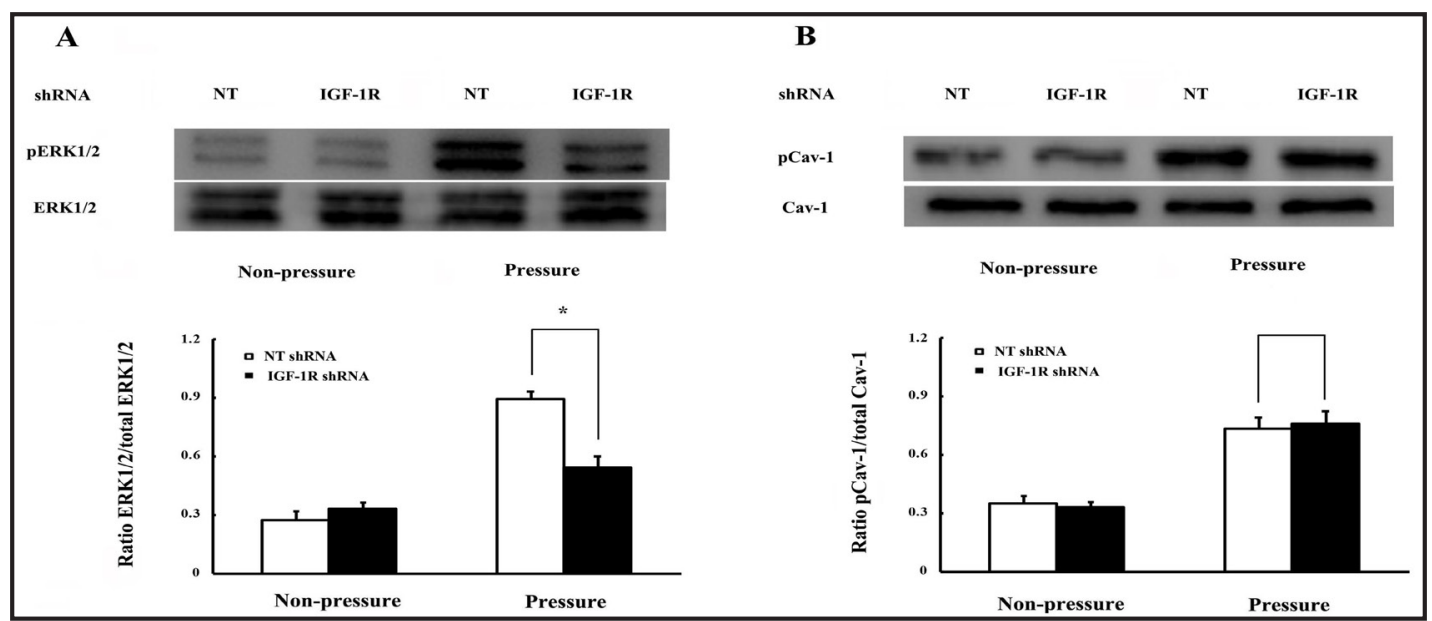

Fig. 5. Effects of IGF-1R on the expression and phosphorylation of Cav-1 and ERK1/2 under conditions of periodic mechanical stress (IGF-1R shRNA)After pretreatment with control or IGF-1R shRNA, rat chondrocytes were cultured in vitro for $1 \mathrm{~h}$ with or without periodic mechanical stress. The expression and phosphorylation levels of Cav-1 and ERK1/2 were detected by Western blotting. The total amount of each protein served as a control. Gray values are represented with a histogram $n=5,{ }^{*} \mathrm{P}<0.05$ for each). The aforementioned images are representative results of Western blotting. The phosphorylation levels of Cav-1$\mathrm{Tyr}^{14}$ and ERK1/2-Thr ${ }^{202} / \mathrm{Tyr}^{204}$ in the IGF-1R shRNA pretreatment group significantly decreased relative to those of the control group under conditions of periodic mechanical stress ( $n=5, P<0.05$ for each; Student's unpaired $t$ test), whereas the activation of Cav-1-Tyr ${ }^{14}$ in the IGF-1R shRNA pretreatment group was not affected ( $n=5, P>0.05$ for each; Student's unpaired t test).

\section{KARGER}




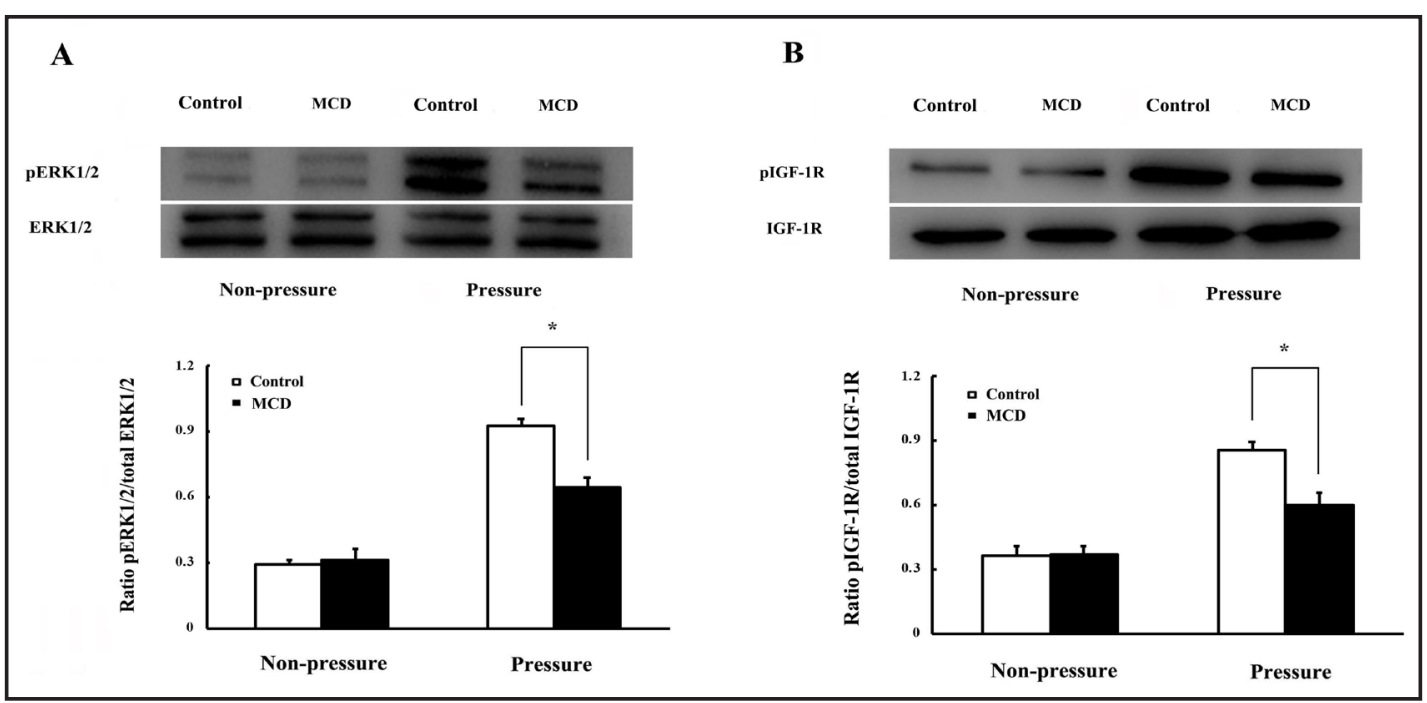

Fig. 6. Effects of Cav-1 on the expression and phosphorylation of IGF-1R and ERK1/2 under conditions of periodic mechanical stress (MCD)After pretreatment with DMSO or Cav-1 inhibitor MCD, rat chondrocytes were cultured in vitro for $1 \mathrm{~h}$ with or without periodic mechanical stress. The expression and phosphorylation levels of IGF-1R, and ERK1/2 were detected by Western blotting. The total amount of each protein served as a control. Gray values are represented with a histogram ( $\mathrm{n}=5,{ }^{*} \mathrm{P}<0.05$ for each). The aforementioned images are representative results of Western blotting. The phosphorylation levels of IGF-1R-Tyr ${ }^{1135 / 1136}$ and ERK1/2-Thr ${ }^{202} / \mathrm{Tyr}^{204}$ in the Cav-1 selective inhibitor pretreatment group significantly decreased relative to those of the control group under conditions of periodic mechanical stress ( $n=5, P<0.05$ for each; Student's unpaired $t$ test).

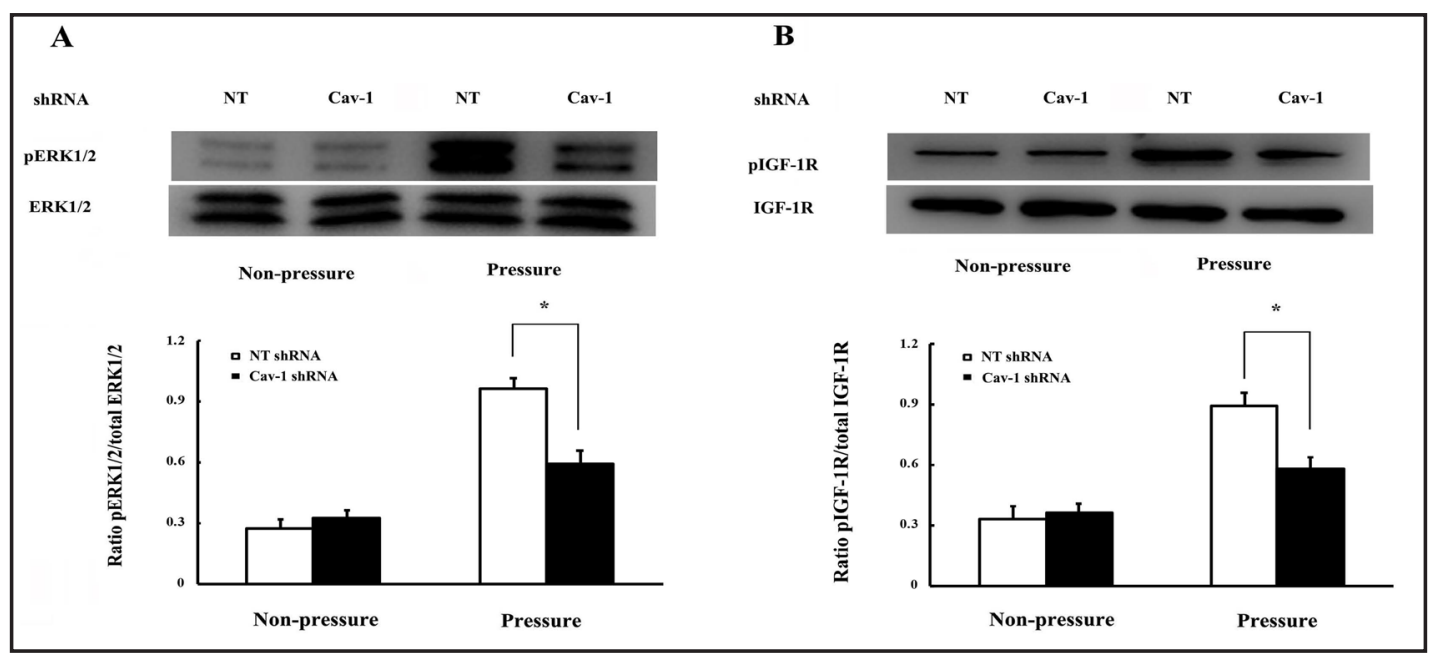

Fig. 7. Effects of Cav-1 on the expression and phosphorylation of IGF-1R and ERK1/2 under conditions of periodic mechanical stress (Cav-1 shRNA)After pretreatment with control or Cav-1 shRNA, rat chondrocytes were cultured in vitro for $1 \mathrm{~h}$ with or without periodic mechanical stress. The expression and phosphorylation levels of IGF-1R and ERK1/2 were detected by Western blotting. The total amount of each protein served as a control. Gray values are represented with a histogram ( $\mathrm{n}=5$, ${ }^{*} \mathrm{P}<0.05$ for each). The aforementioned images are representative results of Western blotting. The phosphorylation levels of IGF-1R-Tyr ${ }^{1135 / 1136}$ and ERK1/2-Thr ${ }^{202} / \mathrm{Tyr}^{204}$ in the Cav-1 shRNA pretreatment group significantly decreased relative to those of the control group under conditions of periodic mechanical stress ( $\mathrm{n}=5, \mathrm{P}<0.05$ for each; Student's unpaired t test). 
IGF-1R is a member of a larger superfamily of receptor kinases, and could sense and respond to mechanical stimuli in several nonchondrocytic cell types $[6,25,26]$. This study investigated and considered IGF-1R signals to be candidate signals for inducing chondrocyte proliferation by periodic mechanical stress. We observed that periodic mechanical stress induces significantly increased phosphorylation of IGF-1R at Tyr ${ }^{1135 / 1136}$ in relation to that under static conditions in chondrocytes. Pretreatment with the selective inhibitors of IGF-1R Linsitinib and shRNA targeted to IGF-1R significantly reduced chondrocyte proliferation under mechanical stress. This was the first study on the mechanosensing role of IGF-1R in chondrocytes. And these results clearly highlighted the significance of IGF-1R in chondrocytic adaption to physical mechanical stimulation. However, this may not be universally true. Despite the importance of IGF-1R in mediating chondrocyte proliferation, it is possible that other growth factor receptors may also play an indispensable role under conditions of mechanical stimuli.

The phosphorylation sites have been identified in the intracellular domain of IGF$1 \mathrm{R}$, which allow for specific binding and modulation of multiple downstream effectors $[27,28]$. An earlier study by Wang et al. confirmed that low shear stress-induced IGF-1R phosphorylation modulates smooth muscle cell behavior and functions (proliferation and phenotype) via enhancing Akt activation [29]. In the present study, the activation of ERK1/2 was found to be attenuated as well when the activity of IGF-1R was inhibited by Linsitinib or shRNA targeted to IGF-1R, suggesting that IGF-1R activation of ERK1/2 contributes to the mechanism by which chondrocytes sense and respond to periodic mechanical stress. Our present observation seems consistent with the reports of IGF-1R modulations and interactions with ERK1/2 in other cell types [30,31]. However, this relationship has not been investigated in chondrocytes under mechanical stimulation. Hence, it could be concluded that IGF-1R mediated at least some mitogenic mechanotransduced signals through ERK1/2 in chondrocytes, and do not exclude a possible role for other signal proteins downstream of IGF-1R.

Cav-1 is generally viewed as an important non-integrin mechanosensor that transmits mechanical stimuli from extracellular matrix into intracellular biochemical signals $[11,32]$. The present study showed that periodic mechanical stress induces significantly increased phosphorylation of Cav-1 at $\mathrm{Tyr}^{14}$ in relation to that under static conditions in chondrocytes. And the specific Cav-1 inhibitor MCD and shRNA targeted to Cav-1 blocked chondrocyte proliferation in response to periodic mechanical stress. To the best of our knowledge, this is the first study to focus on the significance of mechanosensor Cav-1 in chondrocyte signaling under periodic mechanical stress. These results provide definitive evidence to support the participation of Cav-1 signaling in the periodic mechanical stress-initiated mitogenic response in chondrocytes.

Increasing evidence showed that Cav-1 activation is essential for several downstream transduction signal events $[33,34]$. The activation of ERK1/2 by Cav- 1 has recently been demonstrated to be a signaling transduction mechanism in human 1321N1 astrocytoma cells [35]. The present study found that inhibition of Cav-1 with MCD and shRNA targeted to Cav-1 prevented the activation of ERK1/2, suggesting functional links between Cav-1 and 
ERK1/2 signaling. The coincidence of Cav-1 with IGF-1R, as nonintegrin mechanosensors and upstream mediators of ERK1/2 modulation of chondrocytic mitogenic effects, raises the possibility that there may be some causal relationship between Cav-1 and IGF-1R. Cav1 has been shown to be required for activation of growth factor receptors, including IGF$1 \mathrm{R}$ in many nonchondrocytic cell types subjected to several stimuli in different cell culture systems in diverse complex signaling pathways $[19,36]$. Consistent with our conjecture, the inhibition of cyclic mechanical stress-stimulated IGF-1R by MCD or shRNA targeted to Cav-1, instead of being activated by an IGF-1R-dependent mechanism, suggested that Cav-1 might be responsible for the initial IGF-1R activation in the present study. These data above support the concept that Cav-1 might be a proximal kinase in the signal pathway that leads, through IGF-1R, to the increased ERK1/2 activation and chondrocyte proliferation in response to periodic mechanical stress.

In conclusion, the present study found that periodic mechanical stress could stimulate the phosphorylation of Cav-1, which in turn activated IGF-1R. Activated IGF-1R led to ERK1/2 mitogenic signaling cascade in chondrocytes. Thus, Cav-1-IGF-1R-ERK1/2 constituted at least one critical signal transduction pathway for chondrocyte proliferation induced by periodic mechanical stress (Fig.8). These results of our previous studies and the present study provide a stronger viewpoint for further investigations into chondrocyte mechanobiology under periodic mechanical stress and the way to improve the quality of tissue-engineered cartilage.

\section{Acknowledgements}

This work was supported by the National Natural and Science Foundation (81501874), Jiangsu Province Health and Family Planning Commission Foundation (Q201511 and QNRC2016139) (all foundations to Kewei Ren); The Project of Invigorating Health Care through Science, Technology and Education (Jiangsu Provincial Medical Youth Talent), Changzhou High-level Medical Talents Training Project (2016CZBJ029), Jiangsu planned projects for Postdoctoral Research Funds (1701001A) and Changzhou International Scientific and Technology Cooperation Project (CZ20170021) (all foundations to Luming Nong).

\section{Disclosure Statement}

The authors declare that they have no competing interests.

\section{References}

1 Liang W, Zhu C, Liu F, Cui W, Wang Q, Chen Z, Zhou Q, Xu S, Zhai C, Fan W: Integrin beta1 gene therapy enhances in vitro creation of tissue-engineered cartilage under periodic mechanical stress. Cell Physiol Biochem 2015;37:1301-1314.

-2 Li Z, Wang Z, Xu S, Liang W, Fan W: Proteomic analysis reveals a new benefit of periodic mechanical stress on chondrocytes. Cell Physiol Biochem 2017;44:1578-1590.

- Ali M, Kumar A, Pandey BN: Thorium induced cytoproliferative effect in human liver cell hepg2: Role of insulin-like growth factor 1 receptor and downstream signaling. Chem Biol Interact 2014;211:29-35.

4 Rao W, Li H, Song F, Zhang R, Yin Q, Wang Y, Xi Y, Ge H: Ova66 increases cell growth, invasion and survival via regulation of igf-1r-mapk signaling in human cancer cells. Carcinogenesis 2014;35:1573-1581.

5 Ma Y, Fu S, Lu L, Wang X: Role of androgen receptor on cyclic mechanical stretch-regulated proliferation of c2c12 myoblasts and its upstream signals: Igf-1-mediated pi3k/akt and mapks pathways. Mol Cell Endocrinol 2017;450:83-93. 


\section{Cellular Physiology Cell Physiol Biochem 2018;48:1652-1663

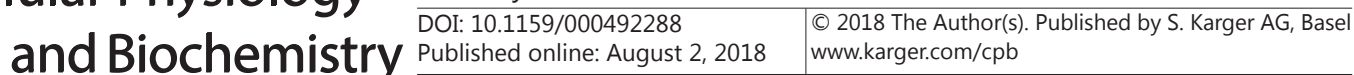

6 Kubota T, Elalieh HZ, Saless N, Fong C, Wang Y, Babey M, Cheng Z, Bikle DD: Insulin-like growth factor-1 receptor in mature osteoblasts is required for periosteal bone formation induced by reloading. Acta Astronaut 2013;92:73-78.

7 Fu S, Yin L, Lin X, Lu J, Wang X: Effects of cyclic mechanical stretch on the proliferation of 16 myoblasts and its mechanisms: Pi3k/akt and mapk signal pathways regulated by igf-1 receptor. Int J Mol Sci 2018;19.

8 Chen Y, Ke J, Long X, Meng Q Deng M, Fang W, Li J, Cai H, Chen S: Insulin-like growth factor-1 boosts the developing process of condylar hyperplasia by stimulating chondrocytes proliferation. Osteoarthritis Cartilage 2012;20:279-287.

-9 Faggi F, Chiarelli N, Colombi M, Mitola S, Ronca R, Madaro L, Bouche M, Poliani PL, Vezzoli M, Longhena F, Monti E, Salani B, Maggi D, Keller C, Fanzani A: Cavin-1 and caveolin-1 are both required to support cell proliferation, migration and anchorage-independent cell growth in rhabdomyosarcoma. Lab Invest 2015;95:585-602.

10 Campbell L, Al-Jayyoussi G, Gutteridge R, Gumbleton N, Griffiths R, Gumbleton S, Smith MW, Griffiths DF, Gumbleton M: Caveolin-1 in renal cell carcinoma promotes tumour cell invasion, and in co-operation with perk predicts metastases in patients with clinically confined disease. J Transl Med 2013;11:255.

11 Goedicke-Fritz S, Kaistha A, Kacik M, Markert S, Hofmeister A, Busch C, Banfer S, Jacob R, Grgic I, Hoyer J: Evidence for functional and dynamic microcompartmentation of cav- $1 / \operatorname{trpv} 4 / \mathrm{k}$ (ca) in caveolae of endothelial cells. Eur J Cell Biol 2015;94:391-400.

-12 Elliott MH, Ashpole NE, Gu X, Herrnberger L, McClellan ME, Griffith GL, Reagan AM, Boyce TM, Tanito M, Tamm ER, Stamer WD: Caveolin-1 modulates intraocular pressure: Implications for caveolae mechanoprotection in glaucoma. Sci Rep 2016;6:37127.

13 Xiong N, Li S, Tang K, Bai H, Peng Y, Yang H, Wu C, Liu Y: Involvement of caveolin-1 in low shear stressinduced breast cancer cell motility and adhesion: Roles of fak/src and rock/p-mlc pathways. Biochim Biophys Acta 2017;1864:12-22.

14 Chai Q, Wang XL, Zeldin DC, Lee HC: Role of caveolae in shear stress-mediated endothelium-dependent dilation in coronary arteries. Cardiovasc Res 2013;100:151-159.

15 Yang H, Guan L, Li S, Jiang Y, Xiong N, Li L, Wu C, Zeng H, Liu Y: Mechanosensitive caveolin-1 activationinduced pi3k/akt/mtor signaling pathway promotes breast cancer motility, invadopodia formation and metastasis in vivo. Oncotarget 2016;7:16227-16247.

16 Lal H, Verma SK, Feng H, Golden HB, Gerilechaogetu F, Nizamutdinov D, Foster DM, Glaser SS, Dostal DE: Caveolin and beta1-integrin coordinate angiotensinogen expression in cardiac myocytes. Int J Cardiol 2013;168:436-445.

17 Zou M, Li Y, Xia S, Chu Q Xiao X, Qiu H, Chen Y, Zheng Z, Liu F, Zhuang L, Yu S: Knockdown of caveolin-1 sensitizes human basal-like triple-negative breast cancer cells to radiation. Cell Physiol Biochem 2017;44:778-791.

18 Zhang B, Peng F, Wu D, Ingram AJ, Gao B, Krepinsky JC: Caveolin-1 phosphorylation is required for stretchinduced egfr and akt activation in mesangial cells. Cell Signal 2007;19:1690-1700.

19 Yang SJ, Chen CY, Chang GD, Wen HC, Chang SC, Liao JF, Chang CH: Activation of akt by advanced glycation end products (ages): Involvement of igf-1 receptor and caveolin-1. PLoS One 2013;8:e58100.

-20 He P, Shen N, Gao G, Jiang X, Sun H, Zhou D, Xu N, Nong L, Ren K: Periodic mechanical stress activates pkcdelta-dependent egfr mitogenic signals in rat chondrocytes via pi3k-akt and erk1/2. Cell Physiol Biochem 2016;39:1281-1294.

21 Liang W, Li Z, Wang Z, Zhou J, Song H, Xu S, Cui W, Wang Q Chen Z, Liu F, Fan W: Periodic mechanical stress induces chondrocyte proliferation and matrix synthesis via camkii-mediated pyk2 signaling. Cell Physiol Biochem 2017;42:383-396.

22 Yue HT, Fan WM, Ma YM: Tissue engineered cartilage in response to diferent cyclic pressure.Xpression in a pkc- andrho kinase-dependent manner. Chin J Orthop Trauma 2007;9:661-664.

23 Zhang GC, Fan WM: Effects of cyclic pressure at different frequendes on the construction of tissueengineering cartilage. Chin J Exp Surg 2007;24:677-678.

24 Xu S, Li Z, Wang Z, Zhai C, Liang W, Zhu C, Fan W: Proteomic analysis reveals grb2 as a key regulator of periodic mechanical stress transduction in chondrocytes. Cell Physiol Biochem 2017;44:1509-1525.

25 Tian F, Wang Y, Bikle DD: Igf-1 signaling mediated cell-specific skeletal mechano-transduction. J Orthop Res 2018;36:576-583. 


\section{Cellular Physiology Cell Physiol Biochem 2018;48:1652-1663

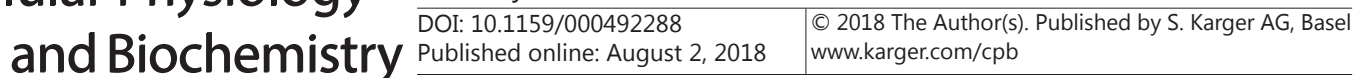

Ren et al.: Cav-1-Dependent IGF-1R Mitogenic Signals Through ERK1/2

26 Santoro M, Lamhamedi-Cherradi SE, Menegaz BA, Ludwig JA, Mikos AG: Flow perfusion effects on threedimensional culture and drug sensitivity of ewing sarcoma. Proc Natl Acad Sci U S A 2015;112:1030410309.

27 Zhang Y, Qin W, Qian Z, Liu X, Wang H, Gong S, Sun YG, Snutch TP, Jiang X, Tao J: Peripheral pain is enhanced by insulin-like growth factor 1 through a g protein-mediated stimulation of t-type calcium channels. Sci Signal 2014;7:ra94.

28 Cho YL, Hur SM, Kim JY, Kim JH, Lee DK, Choe J, Won MH, Ha KS, Jeoung D, Han S, Ryoo S, Lee H, Min JK, Kwon YG, Kim DH, Kim YM: Specific activation of insulin-like growth factor-1 receptor by ginsenoside rg5 promotes angiogenesis and vasorelaxation. J Biol Chem 2015;290:467-477.

29 Wang L, Han Y, Shen Y, Yan ZQ, Zhang P, Yao QP, Shen BR, Gao LZ, Qi YX, Jiang ZL: Endothelial insulin-like growth factor-1 modulates proliferation and phenotype of smooth muscle cells induced by low shear stress. Ann Biomed Eng 2014;42:776-786.

30 Jiang G, Wang W, Cao Q Gu J, Mi X, Wang K, Chen G, Wang X: Insulin growth factor-1 (igf-1) enhances hippocampal excitatory and seizure activity through igf-1 receptor-mediated mechanisms in the epileptic brain. Clin Sci (Lond) 2015;129:1047-1060.

-31 Wang H, Qin J, Gong S, Feng B, Zhang Y, Tao J: Insulin-like growth factor-1 receptor-mediated inhibition of a-type $\mathrm{k}(+)$ current induces sensory neuronal hyperexcitability through the phosphatidylinositol 3-kinase and extracellular signal-regulated kinase 1/2 pathways, independently of akt. Endocrinology 2014;155:168-179.

-32 Soares ES, Mendonca MCP, da Cruz-Hofling MA: Caveolae as a target for phoneutria nigriventer spider venom. Neurotoxicology 2016;54:111-118.

-33 Zeng W, Tang J, Li H, Xu H, Lu H, Peng H, Lin C, Gao R, Lin S, Lin K, Liu K, Jiang Y, Weng J, Zeng L: Caveolin-1 deficiency protects pancreatic beta cells against palmitate-induced dysfunction and apoptosis. Cell Signal 2018;47:65-78.

-34 Wang W, Gu L, Verkhratsky A, Peng L: Ammonium increases trpc1 expression via cav-1/pten/akt/gsk3beta pathway. Neurochem Res 2017;42:762-776.

35 Martinez NA, Ayala AM, Martinez M, Martinez-Rivera FJ, Miranda JD, Silva WI: Caveolin-1 regulates the p2y2 receptor signaling in human 1321n1 astrocytoma cells. J Biol Chem 2016;291:12208-12222.

36 Luan TY, Zhu TN, Cui YJ, Zhang G, Song XJ, Gao DM, Zhang YM, Zhao QL, Liu S, Su TY, Zhao RJ: Expression of caveolin-1 is correlated with lung adenocarcinoma proliferation, migration, and invasion. Med Oncol 2015;32:207. 